\title{
Patrones mastográficos en las mujeres mexicanas
}

\section{Mammography patterns in Mexican women}

Selene T. Mancilla-Mazariegos ${ }^{1 *}$ y Carolina González-Vergara ${ }^{2}$

${ }^{1}$ Facultad Mexicana de Medicina, Universidad La Salle; ${ }^{2}$ División de Educación Médica, Hospital Ángeles Mocel. Ciudad de México, México

\section{RESUMEN}

Introducción: La imagen mastográfica es el reflejo de la anatomía mamaria normal y de las alteraciones provocadas por sus procesos patológicos, y dependerá de la proporción que guarden entre sí sus componentes. Actualmente, la clasificación de los patrones mastográficos más empleada es la clasificación BI-RADS ${ }^{\circledR}$ (Breast Imaging Reporting and Data System); sin embargo, su distribución solo ha sido descrita en población norteamericana y no se considera a las mujeres latinas.

Objetivo: Conocer la prevalencia de las densidades mamarias en mujeres mexicanas y comprobar si los porcentajes descritos por BI-RADS ${ }^{\circledR}$ son aplicables a nuestra población.

Método: Se evaluaron 2,000 mujeres entre 40 y 81 años, con mastografía digital diagnóstica o de escrutinio, en el periodo 2013-2015. Las mastografías fueron valoradas por un radiólogo certificado con calificación agregada en mama, utilizando la clasificación de la quinta edición BI-RADS ${ }^{\circledR}$ para categorizar el patrón mastográfico.

Resultados: Se analizó la frecuencia de los cuatro patrones mastográficos, con una distribución global para la muestra de las 2,000 mujeres con los siguientes resultados: 226 con patrón tipo A (11.3\%), 1,296 con patrón tipo B (64.8\%), 400 con patrón tipo C (20\%) y 78 con patrón tipo D $(3.9 \%)$.

Conclusiones: Encontramos que la densidad mamaria predominante en nuestra población corresponde al tipo fibroglandular disperso, y al comparar con los porcentajes descritos por BI-RADS ${ }^{\circledR}$, así como en otros estudios realizados en mujeres estadounidenses, 
concluimos que no son aplicables a nuestra población, ya que los factores asociados a la densidad mamaria son distintos.

Palabras clave: Mamografía. Densidad mamaria. Cáncer de mama.

\section{ABSTRACT}

Introduction: Mammography is the reflection of normal breast anatomy and disturbances caused by pathologic processes, and will depend on the proportion of its components. Currently, the most used classification of mammographic patterns is the BI-RADS ${ }^{\circledR}$ classification, however its pattern distribution has only been described in North American population and women from Latin America have not been considered.

Objective: To learn the prevalence of breast density in Mexican women and confirm whether the rates described in $\mathrm{BI}-\mathrm{RADS}^{\circledR}$ are applicable to our population.

Material and method: 2,000 women between 40 and 81 years of age were evaluated with diagnostic or screening digital mammography in the Hospital from 2013 -2015. Mammographies were read by a certified radiologist with additional qualification in breast reading, using the fifth edition of the BI-RADS ${ }^{\circledR}$ classification to establish the category of the mammographic pattern.

Result: The frequency of the 4 mammographic patterns was analyzed, with an overall distribution for the 2,000 women sample revealing the following results: 226 with Type A pattern (11.3\%), 1,296 with Type B pattern (64.8\%), 400 with Type C pattern (20\%), 78 with Type D pattern (3.9\%).

Conclusions: We found that the predominant breast density in our population is the scattered fibroglandular pattern, and when compared to the rates described by BI-RADS ${ }^{\circledR}$, as well as other studies from US female population, we conclude that they are not applicable to our own population since the factors associated to breast density are different.

Key words: Mammography. Breast density. Breast cancer.

\section{INTRODUCCIÓN}

La imagen mastográfica es el reflejo de la anatomía mamaria normal y de las alteraciones provocadas por sus procesos patológicos. La apariencia de la imagen dependerá de la proporción que guarden entre sí sus componentes estructurales, que de acuerdo con las descripciones de Tabár, et al. ${ }^{1}$ son cuatro elementos descritos como densidades nodulares, lineales, homogéneas carentes de estructura y zonas radiotransparentes, las cuales pueden 
distribuirse de manera aleatoria o regionalmente dentro de una misma mama, y comportarse de forma similar o diferente en cada mama en una misma paciente, y a su vez con una amplia variabilidad entre pacientes, por lo que se clasificaron en cinco patrones:

- Tipo I: los cuatro componentes están presentes en una proporción similar.

- Tipo II: predominio de tejido fibroadiposo y densidades lineales.

- Tipo III: similar al tipo II, pero con ductos prominentes retroareolares.

- Tipo IV: predominio de las densidades nodulares y lineales.

- Tipo V: predominio del tejido fibroso homogéneo carente de estructura ${ }^{1}$.

Dicha variabilidad de los patrones se debe a que, a lo largo de la vida de la mujer, el tejido mamario presenta diversas modificaciones secundarias a la exposición hormonal endógena que recibe, iniciando en la pubertad con el desarrollo de lóbulos, lobulillos y conductos galactóforos ${ }^{2}$.

Posteriormente, en la menarca, los estrógenos se encargan de la proliferación celular, y en conjunto con la progesterona incrementan este proceso, lo que lleva a la formación de la estructura alveolo-lobulillar que condiciona que la mama se torne más voluminosa y con mayor contenido de agua, lo cual se reflejaría en la mamografía con una mayor densidad ${ }^{3}$.

Aproximadamente a los 45 años, cuando la mujer se encuentra en la perimenopausia, el estímulo hormonal comienza a descender hasta la menopausia, que es alrededor de los 55 años, y se dejan de presentar cambios cíclicos hormonales, lo que provoca que el tejido lobular involucione, mientras que las porciones proximales del sistema ductal permanecen intactas, dando una apariencia mamográfica más radiotransparente por el reemplazo de tejido graso ${ }^{3}$.

En el caso de las mujeres que se someten a terapia de reemplazo hormonal, estos agentes exógenos van a provocar efectos semejantes a los de las hormonas de producción endógena, dando como resultado que en la mamografía se presente un incremento en la densidad de distribución focal, multifocal o difusa, así como zonas de asimetría ${ }^{2,3}$.

Debido a todos estos cambios, es esencial el papel de la mamografía, ya que posee una sensibilidad del $80-85 \%$ para la detección de cáncer; sin embargo, en los casos en que el patrón mamográfico es heterogéneamente denso o sumamente denso, el diagnóstico se puede tornar complicado, al no visualizar lesiones que pueden permanecer ocultas hasta que alcanzan un mayor tamaño, lo que repercute en un diagnóstico tardío y una etapa clínica avanzada, aumentado la morbimortalidad ${ }^{4}$.

En las mujeres con tejido mamario denso, el riesgo de presentar cáncer de mama se describe con un incremento de 4-6 veces más en comparación con aquellas mujeres con tejido mamario no denso, y aunque la densidad por sí misma se considera un factor de riesgo moderado, no significa que se le deba restar importancia ${ }^{5,6}$. 

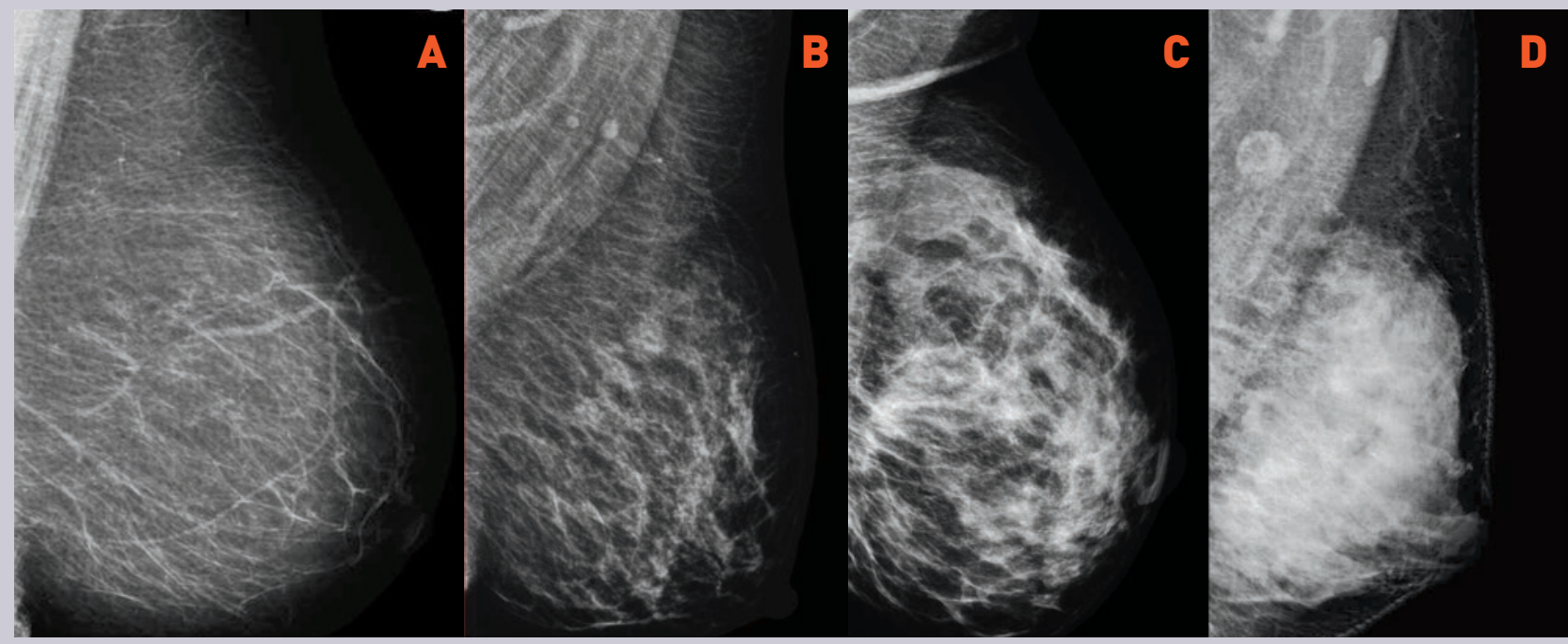

Figura 1. Imágenes de PACS del hospital. Mastografías digitales en proyecciones medio-oblicuo laterales, en las que se observan los cuatro patrones mastográficos de acuerdo con BI-RADS ${ }^{\circledR}$ 2013. A: predominantemente graso. B: fibroglandular disperso. C: heterogéneamente denso. D: extremadamente denso.

Cuando el riesgo se correlaciona con la densidad mamaria, se ha observado que para las mujeres con mamas heterogéneamente densas (40\% de la población) es alrededor de 1-2 veces mayor, mientras que para las mujeres con mamas sumamente densas $(10 \%$ de la población) el riesgo es 2 veces mayor?

A lo largo del tiempo se han desarrollado varias clasificaciones de las densidades mastográficas, todas ellas subjetivas; la primera fue la descrita por Wolfe en 1976. Actualmente, el método más utilizado en México es el propuesto por el American College of Radiology, que es la clasificación BI-RADS ${ }^{\circledR}$ (Breast Imaging Reporting and Data System), la cual cataloga la densidad mamaria en cuatro patrones: el tipo A para mamas enteramente grasas, el tipo B para tejido fibroglandular disperso, el tipo $C$ para tejido mamario heterogéneamente denso, y el tipo D para tejido mamario extremadamente denso. En sus inicios tenía un enfoque cualitativo y posteriormente se centró en porcentajes de tejido fibroglandular ${ }^{4,6}$. La quinta edición de BI-RADS ${ }^{\circledR}$, en 2013, no aconseja la utilización de porcentajes y considera más importante la posibilidad de que una lesión quede «oculta» por tejido denso, lo cual dificultaría su diagnóstico. Por consenso, se consideran como mamas densas las categorías C y D, y como mamas no densas las categorías A y B. La distribución de estas categorías en la población norteamericana es de aproximadamente el $10 \%$ para el tipo A, el $40 \%$ para el tipo B, el $40 \%$ para el tipo C y el $10 \%$ para el tipo $\mathrm{D}^{4,6}$ (Fig. 1); sin embargo, estos porcentajes referidos en la literatura no consideran a las mujeres latinas, motivo por el cual surge la necesidad de conocer la prevalencia de las densidades mamarias en las mujeres mexicanas.

El objetivo del presente estudio fue conocer la prevalencia de las densidades mamarias 
en las mujeres mexicanas, y comprobar si los porcentajes descritos por BI-RADS ${ }^{\circledR}$ son aplicables a nuestra población.

\section{MÉTODO}

Se estudió una población de 2,000 mujeres mexicanas entre 40 y 81 años (con una media de 55.55 años), a las cuales se les realizó mastografía digital diagnóstica o de escrutinio en el hospital durante el periodo de enero de 2013 a diciembre de 2015.

Se realizó un estudio observacional, analítico y transversal para recabar datos estadísticos que sustenten si los porcentajes descritos por el BI-RADS ${ }^{\circledR}$ son aplicables a nuestra población.

A cada paciente se le tomaron tres proyecciones bilaterales: cráneo-caudal, medio-lateral oblicua y cráneo-caudal extrema; y fueron valoradas por un radiólogo certificado con calificación agregada en mama y acreditado por el Consejo Mexicano de Radiología e Imagen, utilizando la clasificación de la quinta edición BI-RADS ${ }^{\circledR}$ para categorizar el patrón mastográfico.

La adquisición se realizó con equipo de mastografía digital de campo completo Giotto Image 3D, modelo 2011/04, por un técnico capacitado en la toma de mastografías.

\section{RESULTADOS}

Una vez obtenida la muestra, las mujeres se dividieron en cuatro grupos de edad (40-50, 51-60, 61-70 y 71-81 años), encontrándose que en el grupo de 40 a 50 años hubo 36 pacientes
TABla 1. Patrones mastográficos. Distribución por grupos de edad en 2,000 mujeres mexicanas de 40-81 años

\begin{tabular}{|c|c|c|}
\hline Grupo de edad & N. ${ }^{0}$ pacientes & Patrón mastográfico \\
\hline \multirow[t]{4}{*}{ 40-50 años } & 36 & $A$ \\
\hline & 377 & B \\
\hline & 273 & C \\
\hline & 59 & D \\
\hline \multirow[t]{4}{*}{ 51-60 años } & 70 & $A$ \\
\hline & 416 & B \\
\hline & 87 & C \\
\hline & 11 & D \\
\hline \multirow[t]{4}{*}{ 61-70 años } & 86 & $A$ \\
\hline & 341 & B \\
\hline & 25 & C \\
\hline & 4 & D \\
\hline \multirow[t]{4}{*}{ 71-81 años } & 34 & $A$ \\
\hline & 161 & B \\
\hline & 15 & C \\
\hline & 4 & D \\
\hline
\end{tabular}

que presentaron patrón tipo A (5\%), 377 con patrón tipo B $(50 \%), 273$ con patrón tipo C (37\%) y 59 con patrón tipo D (8\%). En el grupo de 51 a 60 años, 70 presentaron patrón tipo A (12\%), 416 patrón tipo B $(71 \%), 87$ patrón tipo C (15\%) y 11 patrón tipo D (2\%). En el grupo de 61 a 71 años hubo 86 con patrón tipo A (19\%), 341 con patrón tipo B (75\%), 25 con patrón tipo C $(5 \%)$ y 4 con patrón tipo D (1\%). En el grupo de 71 a 81 años, 34 presentaron patrón tipo A (16\%), 161 patrón tipo B $(75 \%)$, 15 patrón tipo C $(7 \%)$ y 4 patrón tipo $\mathrm{D}(2 \%)$ (Tabla 1). Por lo tanto, la distribución global para la muestra de las 2,000 mujeres es la siguiente: 226 con patrón tipo A (11.3\%), 1,296 con patrón tipo B (64.8\%), 400 con patrón tipo C (20\%) y 78 con patrón tipo D (3.9\%) (Fig. 2). 


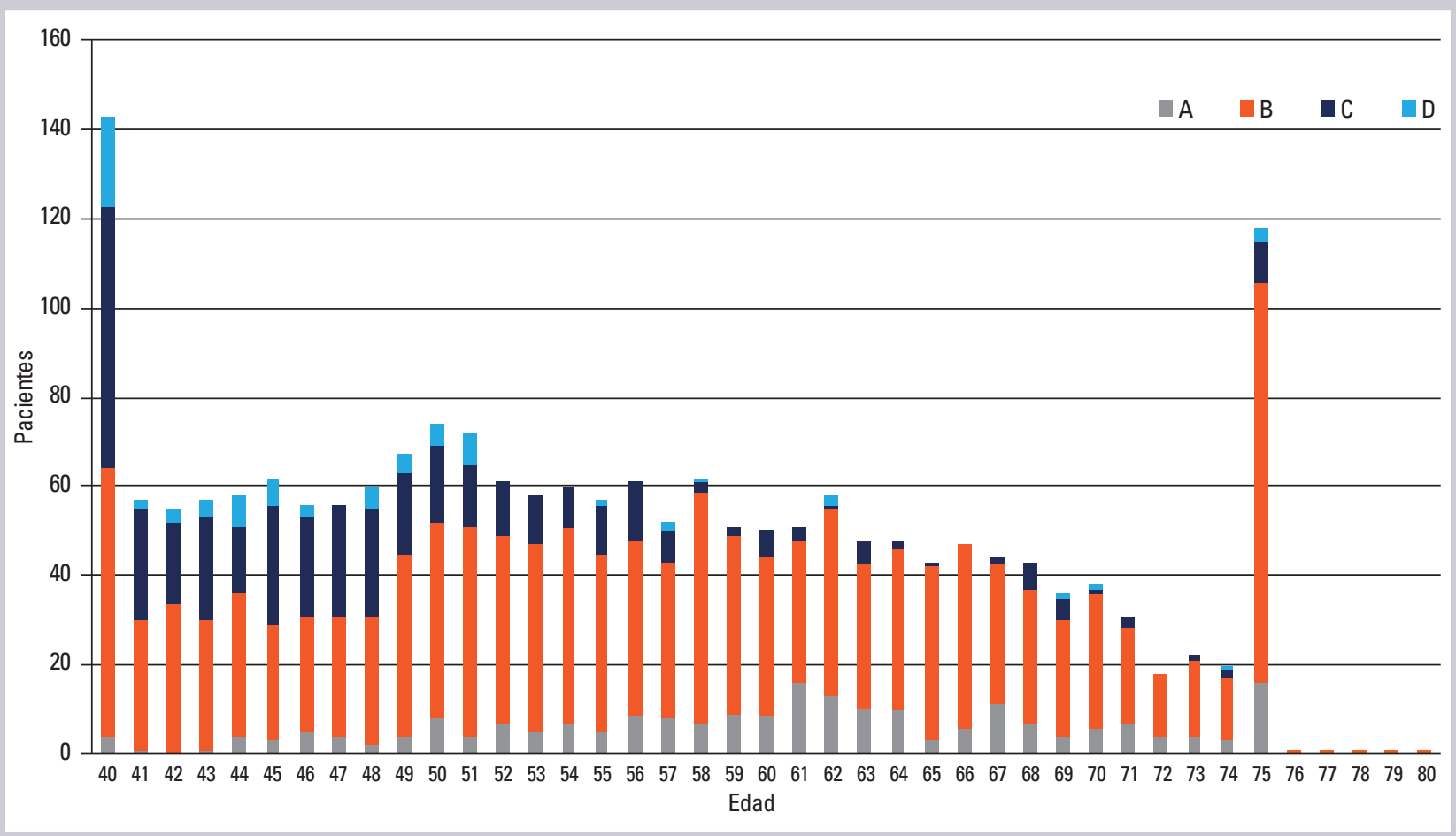

Figura 2. Patrones Mastográficos: distribución por grupos etarios en 2000 mujeres mexicanas de $40-81$ años. $A, B, C, D$, corresponden a los datos de la tabla 2.

\section{DISCUSIÓN}

De acuerdo con los resultados obtenidos al compararlos con los porcentajes de BI-RADS ${ }^{\circledR}$, se evidencia que en las mujeres mexicanas la distribución es totalmente distinta, predominando el patrón fibroglandular disperso (tipo B) en todos los grupos de edad evaluados (Tabla 2).

En un artículo publicado en 2016 por Brian L. Sprague, et al. ${ }^{8}$ se evaluaron las mastografías de 145,123 mujeres de entre 40 y 89 años, de las cuales 5812 eran hispanas, y sus resultados globales reportaron una media del $48.3 \%$ para el patrón tipo B o fibroglandular disperso y del $33.8 \%$ para el patrón C o heterogéneamente denso. Al comparar con BI-RADS ${ }^{\circledR}$ estos porcentajes muestran un predominio
TABLA 2. Distribución global de los patrones mastográficos en 2,000 mujeres mexicanas de 40-81 años de edad

\begin{tabular}{|l|c|c|}
\hline Patrón & N. $^{0}$ casos & Porcentaje \\
\hline A & 226 & $11 \%$ \\
\hline B & 1,296 & $65 \%$ \\
\hline C & 400 & $20 \%$ \\
\hline D & 78 & $4 \%$ \\
\hline Total & 2,000 & $100 \%$ \\
\hline
\end{tabular}

significativo del patrón tipo $\mathrm{B}^{9}$; sin embargo, el estudio no lleva a cabo una comparación de los diferentes grupos étnicos de la muestra que permita analizar si existen diferencias estadísticamente significativas entre ellos.

En el presente estudio se observó que el patrón tipo B es el predominante, por lo que podemos deducir que la sensibilidad de la 
mastografía es elevada para la mayoría de las mujeres mexicanas, lo que permite una mejor detección oportuna de cáncer de mama con menor riesgo asociado a la densidad que en otros tipos de población.

\section{CONCLUSIONES}

Los resultados obtenidos demuestran que la densidad mamaria predominante en la población estudiada corresponde al tipo B o fibroglandular disperso en todos los grupos de edad evaluados, y que al comparar con los porcentajes descritos por BI-RADS ${ }^{\circledR}$, así como con otros estudios realizados en mujeres estadounidenses, concluimos que no son aplicables a nuestra población, ya que los factores asociados a la densidad mamaria son distintos.

Teniendo estos resultados en cuenta podemos inferir que el predominio de este patrón fibroglandular disperso permitirá al radiólogo una mejor visualización de las lesiones mamarias, de tal manera que esto contribuye en un incremento de la detección oportuna del cáncer de mama.
Los resultados de nuestro trabajo abren una ventana de oportunidad a las pacientes mexicanas ante la posibilidad global de tener un diagnóstico oportuno más adecuado directamente relacionado con el patrón mamario general de la población.

\section{BIBLIOGRAFÍA}

1. Tabár L, Tot T, Dean PB. Breast cancer : the art and science of early detection with mammography : perception, interpretation, histopathologic correlation. Stuttgart, New York: Thieme; 2005. p. 16-38.

2. Stines J, Tristant $H$. The normal breast and its variations in mammography. Eur J Radiol. 2005;54:26-36.

3. Abramson RG, Mavi A, Cermik T, Basu S, Wehrli NE, Houseni M, et al. Age-related structural and functional changes in the breast: multimodality correlation with digital mammography, computed tomography, magnetic resonance imaging, and positron emission tomography. Semin Nucl Med. 2007;37:146-53.

4. Al Mousa DS, Ryan EA, Mello-Thoms C, Brennan PC. What effect does mammographic breast density have on lesion detection in digital mammography? Clin Radiol. 2014;69:333-41.

5. Checka CM, Chun JE, Schnabel FR, Lee J, Toth H. The relationship of mammographic density and age: implications for breast cancer screening. Am J Roentgenol. 2012;198:W292-5.

6. Freer PE. Mammographic breast density: impact on breast cancer risk and implications for screening. RadioGraphics. 2015;35:302-15.

7. Gascón I, Casamayor C, Sánchez N, Hernando E, Martínez J, FernándezSanguino AB, et al. Cirugía conservadora en el cáncer de mama. Reconstrucción con malla reabsorbible de poliglactina 910. Rev Senol Patol Mamar. 2017;30:61-7.

8. Sprague BL, Conant EF, Onega T, Garcia MP, Beaber EF, Herschorn SD, et al. Variation in mammographic breast density assessments among radiologists in clinical practice: a multicenter observational study. Ann Intern Med. 2016;165:457-64.

9. Sprague BL, Gangnon RE, Burt V, Trentham-Dietz A, Hampton JM, Wellman RD, et al. Prevalence of mammographically dense breasts in the United States. J Natl Cancer Inst. 2014;106(10). 